PROCEEDINGS OF THE

AMERICAN MATHEMATICAL SOCIETY

Volume 130, Number 8, Pages 2271-2274

S 0002-9939(02)06219-6

Article electronically published on March 15, 2002

\title{
AN ANALYTICITY CRITERION FOR REGULARIZED SEMIGROUPS
}

\author{
QUAN ZHENG AND YONGSHENG ZHAO
}

(Communicated by Joseph A. Ball)

\begin{abstract}
A generalization of Kato's analyticity criterion for $C_{0}$-semigroups to exponentially bounded regularized semigroups is given by using the method of Laplace transforms.
\end{abstract}

The motivation for this note is that Liu 4 and Kantorovitz 2 proved an analyticity criterion for semigroups and contraction semigroups, respectively. In fact, Liu's result was known earlier by Kato [3, p. 492]. On the other hand, regularized semigroups have received much attention since 1987 (see, e.g. [1] and the references therein). In this note, we will generalize Kato's result to exponentially bounded regularized semigroups.

In Kato [3], the analyticity criterion for semigroups was derived from the resolvent growth characterization of generators of analytic semigroups. Other proofs were given by Liu [4] and Kantorovitz [2]. The latter, for example, is based on an exponential formula of semigroups and the use of normal families, while the present proof is based on a characterization of Laplace transforms of abstract analytic functions with growth restrictions $[5]$.

Let $B(X)$ be the set of all bounded linear operators from a Banach space $X$ into itself. By $\mathcal{D}(A)$ and $\mathcal{R}(A)$ we denote the domain and range of a linear operator $A$, respectively. For an injective operator $C$ in $B(X)$, we denote by $\rho_{C}(A):=$ $\{\lambda \in \mathbf{C}: \lambda-A$ is injective and $\mathcal{R}(C) \subset \mathcal{R}(\lambda-A)\}$ its $C$-resolvent set and by $R_{C}(\lambda, A):=(\lambda-A)^{-1} C\left(\lambda \in \rho_{C}(A)\right)$ its $C$-resolvent. Set

$$
\Delta_{\alpha}:=\{\lambda \in \mathbf{C}:|\arg \lambda|<\alpha\} \backslash\{0\}
$$

and

$$
\Delta_{\alpha}^{\prime}=\{\lambda \in \mathbf{C}:|\arg \lambda| \leq \alpha\},
$$

where $0<\alpha \leq \pi$. Moreover, $M_{\beta}$ denotes a constant that depends only on $\beta$.

Definition 1. Let $C$ be an injective operator in $B(X)$. A strongly continuous family $T:[0, \infty) \rightarrow B(X)$ is called an (exponentially bounded) $C$-regularized semigroup if $T(0)=C, T(t+s) C=T(t) T(s)(t, s \geq 0)$, and $\|T(t)\| \leq M e^{\omega t}$

Received by the editors December 14, 1999 and, in revised form, November 1, 2000.

2000 Mathematics Subject Classification. Primary 47D62.

Key words and phrases. Regularized semigroup, analyticity, abstract Laplace transform.

This project was supported by the National Science Foundation of China and the Foundation for Excellent Young Teachers of China. 
$(t \geq 0)$ for some constants $M \geq 0, \omega \in \mathbf{R}$. Its generator, $A$, is defined by

$$
\begin{aligned}
\mathcal{D}(A)= & \left\{x \in X: \lim _{t \downarrow 0}(T(t) x-C x) / t \text { exists and is in } \mathcal{R}(C)\right\}, \\
& A x=C^{-1} \lim _{t \downarrow 0}(T(t) x-C x) / t \text { for } x \in \mathcal{D}(A) .
\end{aligned}
$$

We refer to [1 6] for the following lemma, which will be used to conclude that $A$ generates a $C$-regularized semigroup.

Lemma 1. A linear operator $A$ is the generator of a $C$-regularized semigroup if and only if $A=C^{-1} A C,(\omega, \infty) \subset \rho_{C}(A)$, and there exists a strongly continuous family $\{T(t)\}_{t \geq 0}$ with $\|T(t)\| \leq M e^{\omega t}(t \geq 0)$ for some constants $M \geq 0, \omega \in \mathbf{R}$ such that

$$
R_{C}(\lambda, A)=\int_{0}^{\infty} e^{-\lambda t} T(t) x d t \quad \text { for } \lambda>\omega, x \in X
$$

Definition 2. A $C$-regularized semigroup $\{T(t)\}_{t \geq 0}$ is called an analytic $C$-regularized semigroup if

(a) $t \mapsto T(t)$ can be extended analytically to $\Delta_{\alpha}$ for some $\alpha \in(0, \pi / 2]$;

(b) for every $\beta \in(0, \alpha)$, there exist constants $M_{\beta} \geq 0, \omega \in \mathbf{R}$ such that $\|T(t)\| \leq$ $M_{\beta} e^{\omega \operatorname{Re} t}$ for $t \in \Delta_{\beta}$

(c) $t \mapsto T(t)$ is strongly continuous in $\Delta_{\beta}^{\prime}$ for every $\beta \in(0, \alpha)$.

In this case, we write $(A, T(\cdot)) \in H_{C}(\omega, \alpha)$, where $A$ is the generator of $\{T(t)\}_{t \geq 0}$.

The following lemma can be found in 7], which is a modification of Neubrander's result 5 .

Lemma 2. Let $\omega \in \mathbf{R}, \alpha \in(0, \pi / 2]$ and $F:(\omega, \infty) \rightarrow X$. Then the following statements are equivalent:

(a) $F$ is analytic in $\omega+\Delta_{\alpha+\pi / 2}$, and $\|(\lambda-\omega) F(\lambda)\| \leq M_{\beta}\left(\lambda \in \Delta_{\beta+\pi / 2}\right)$ for every $\beta \in(0, \alpha)$.

(b) There exists an analytic function $h: \Delta_{\alpha} \rightarrow X$ with $\|h(t)\| \leq M_{\beta} e^{\omega \operatorname{Re} t}(t \in$ $\left.\Delta_{\beta}\right)$ for every $\beta \in(0, \alpha)$ such that

$$
F(\lambda)=\int_{0}^{\infty} e^{-\lambda t} h(t) d t \quad \text { for } \lambda>\omega
$$

The main result of this note is the following.

Theorem. Let $\omega \in \mathbf{R}$ and $\alpha \in(0, \pi / 2]$. Then $(A, T(\cdot)) \in H_{C}(\omega, \alpha)$ if and only if the following statements hold:

(a) For every $\theta \in(-\alpha, \alpha), e^{i \theta} A$ generates a $C$-regularized semigroup $\left\{T_{\theta}(t)\right\}_{t \geq 0}$.

(b) For every $\beta \in(0, \alpha)$, there exists a constant $M_{\beta} \geq 0$ such that $\left\|T_{\theta}(t)\right\| \leq$ $M_{\beta} e^{\omega t \cos \theta}$ for $t \geq 0$ and $|\theta| \leq \beta$.

(c) For every $\beta \in(0, \alpha), x \in X, \lim _{t \downarrow 0} \sup _{|\theta| \leq \beta}\left\|T_{\theta}(t) x-C x\right\|=0$.

In the case $\overline{\mathcal{D}(A)}=X,(A, T(\cdot)) \in H_{C}(\omega, \alpha)$ if and only if conditions (a) and (b) are satisfied.

Proof. We assume without loss of generality that $\omega=0$. Otherwise we will replace $(A, T(t)) \in H_{C}(\omega, \alpha)$ by $\left(A_{1}, T_{1}(t)\right) \in H_{C}(0, \alpha)$, where $A_{1}=A-\omega$ and $T_{1}(t)=$ $e^{-\omega t} T(t)\left(t \in \Delta_{\alpha}\right)$. 
" $\Rightarrow$ " For every $\theta \in(-\alpha, \alpha)$, let $T_{\theta}(t)=T\left(e^{i \theta} t\right)(t \geq 0)$, then $\left\{T_{\theta}(t)\right\}_{t \geq 0}$ is a $C$-regularized semigroup and satisfies (b) and (c). It remains to show that $e^{i \theta} A$ is the generator of $\left\{T_{\theta}(t)\right\}_{t \geq 0}$. By Lemma 1 and the properties of Laplace transforms we have that $\Delta_{\pi / 2} \subset \rho_{C}(A)$ and

$$
R_{C}(\lambda, A) x=\int_{0}^{\infty} e^{-\lambda t} T(t) x d t \quad \text { for } \operatorname{Re} \lambda>0, x \in X .
$$

In particular, for $\lambda>0$, we have $\lambda e^{-i \theta} \in \rho_{C}(A)$ and

$$
R_{C}\left(\lambda e^{-i \theta}, A\right) x=\int_{0}^{\infty} \exp \left(-\lambda e^{-i \theta} t\right) T(t) x d t \quad \text { for } x \in X .
$$

It therefore follows from Definition 2 that $(0, \infty) \subset \rho_{C}\left(e^{i \theta} A\right)$ and

$$
\begin{aligned}
R_{C}\left(\lambda, e^{i \theta} A\right) x & =\int_{\Gamma_{\theta}} e^{-\lambda z} T\left(e^{i \theta} z\right) x d z \\
& =\int_{0}^{\infty} e^{-\lambda t} T_{\theta}(t) x d t \quad \text { for } \lambda>0, x \in X,
\end{aligned}
$$

where $\Gamma_{\theta}=\left\{t e^{-i \theta}: t \geq 0\right\}$. Also, by Lemma 1 we conclude that $e^{i \theta} A$ is the generator of $\left\{T_{\theta}(t)\right\}_{t \geq 0}$.

" $\Leftarrow$ " For every $\theta \in(-\alpha, \alpha)$, by Lemma 1 we have that $\Delta_{\pi / 2} \subset \rho_{C}\left(e^{i \theta} A\right)$ and

$$
R_{C}\left(\lambda, e^{i \theta} A\right) x=\int_{0}^{\infty} e^{-\lambda t} T_{\theta}(t) x d t \quad \text { for } \operatorname{Re} \lambda>0, x \in X .
$$

Consequently

$$
\rho_{C}(A) \supset \bigcup_{|\theta|<\alpha}\{\lambda \in \mathbf{C} \backslash\{0\}:-\theta-\pi / 2<\arg \lambda<-\theta+\pi / 2\}=\Delta_{\alpha+\pi / 2}
$$

and, by (स*), $R_{C}(\cdot, A): \Delta_{\alpha+\pi / 2} \rightarrow B(X)$ is analytic. Also, for $\lambda \in \Delta_{\beta+\pi / 2}$ $(0<\beta<\alpha)$, we can choose $|\theta|<\beta$ such that $e^{i \theta} \lambda \in \Delta_{\pi / 2}$ and thus, by (四) and (b),

$$
\left\|R_{C}(\lambda, A)\right\|=\left\|R_{C}\left(e^{i \theta} \lambda, e^{i \theta} A\right)\right\| \leq M_{\beta} /|\lambda| .
$$

Now, by Lemma 2, there exists an analytic function $T: \Delta_{\alpha} \rightarrow B(X)$ with $\|T(t)\| \leq$ $M_{\beta}\left(t \in \Delta_{\beta}\right)$ such that

$$
R_{C}(\lambda, A)=\int_{0}^{\infty} e^{-\lambda t} T(t) d t \quad \text { for } \operatorname{Re} \lambda>0 .
$$

Similarly to the proof of (荬, we obtain that

$$
R_{C}\left(\lambda, e^{i \theta} A\right) x=\int_{0}^{\infty} e^{-\lambda t} T\left(t e^{i \theta}\right) x d t \quad \text { for } \lambda>0 .
$$

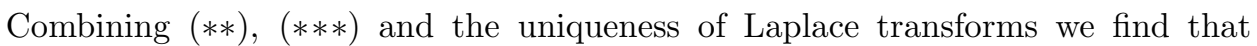
$T\left(t e^{i \theta}\right)=T_{\theta}(t)$ for $t \geq 0$ and $|\theta|<\alpha$, and therefore $(A, T(\cdot)) \in H_{C}(\omega, \alpha)$ follows from conditions $(\mathrm{a})-(\mathrm{c})$.

Finally, if $\overline{\mathcal{D}(A)}=X$, then we only need to show that statements (a) and (b) imply statement (c). In fact, from the proof of the implication " $\Leftarrow$ " and a property 
of regularized semigroups [1, Theorem $3.4(\mathrm{~d})$ ] we deduce that

$$
\begin{aligned}
\lim _{\Delta_{\beta}^{\prime} \ni t \rightarrow 0}\|T(t) x-C x\| & =\lim _{\Delta_{\beta}^{\prime} \ni t \rightarrow 0}\left\|\int_{0}^{|t|} T_{\theta}(s)\left(e^{i \theta} A\right) x d s\right\| \\
& \leq M_{\beta} \lim _{\Delta_{\beta}^{\prime} \ni t \rightarrow 0}|t|\|A x\| \\
& =0 \quad \text { for } x \in \mathcal{D}(A),
\end{aligned}
$$

where $\theta=\arg t$. Since $\overline{\mathcal{D}(A)}=X$, statement (c) follows now from statement (b).

When $\overline{\mathcal{D}(A)}=X$, from Lemma 2 and the proof of the Theorem we have the following Corollary, in which the equivalence of statements (a) and (c) is due to [7]. Corollary 3].

Corollary. Let $\overline{\mathcal{D}(A)}=X, \omega \in \mathbf{R}$ and $\alpha \in(0, \pi / 2]$. Then the following statements are equivalent:

(a) $(A, T(\cdot)) \in H_{C}(\alpha, \omega)$.

(b) $(\omega, \infty) \subset \rho_{C}(A), A=C^{-1} A C$, and there exists an analytic function $T(\cdot)$ : $\Delta_{\alpha} \rightarrow B(X)$ such that $\|T(t)\| \leq M_{\beta} e^{\omega \operatorname{Re} t}\left(t \in \Delta_{\beta}\right)$ for every $\beta \in(0, \alpha)$, and $R_{C}(\lambda, A)=\int_{0}^{\infty} e^{-\lambda t} T(t) d t$ for $\lambda>\omega$.

(c) $\omega+\Delta_{\alpha+\pi / 2} \subset \rho_{C}(A), A=C^{-1} A C$, and $R_{C}(\lambda, A)$ is analytic in $\omega+\Delta_{\alpha+\pi / 2}$ and satisfies $\left\|(\lambda-\omega) R_{C}(\lambda, A)\right\| \leq M_{\beta}\left(\lambda \in \omega+\Delta_{\beta+\pi / 2}\right)$ for every $\beta \in(0, \alpha)$.

\section{ACKNOWLEDGMENT}

We are indebted to the referee for his comments and to the editor for his suggestions.

\section{REFERENCES}

1. R. deLaubenfels, Existence Families, Functional Calculi and Evolution Equations, Lect. Notes in Math., Vol. 1570, Springer-Verlag, Berlin, 1994. MR 96b:47047

2. S. Kantorovitz, On Liu's analyticity criterion for semigroups, Semigroup Forum 53 (1996), 262-265. MR 97d:47042

3. T. Kato, Perturbation Theory for Linear Operators, 2nd. ed., Springer-Verlag, Berlin, 1976. MR 53:11389

4. Y. Liu, An equivalent condition for analytic $C_{0}$-semigroups, J. Math. Anal. Appl. 180 (1993), 71-78. MR 94i:47062

5. F. Neubrander, Abstract elliptic operators, analytic interpolation semigroups and Laplace transforms of analytic functions, Semesterbericht Funktionalanalysis, Tübingen, Wintersemester 15 (1988/89), 163-185.

6. Q. Zheng, Controllability of a class of linear systems in Banach spaces, Proc. Amer. Math. Soc. 123 (1995), 1241-1251. MR 95e:93011

7. Q. Zheng and Y. Lei, Exponentially bounded C-semigroups and integrated semigroups with nondensely defined generators III: Analyticity, Acta Math. Scientia 14 (1994), 107-119. MR 95m:47065

Department of Mathematics, Huazhong University of Science and Technology, Wuhan 430074, People's Republic of China

Department of Mathematics, Huazhong University of Science and Technology, Wuhan 430074, People's Republic of China 